\title{
Graham Priest
}

\section{Metatheory and dialetheism}

\author{
Graham Priest \\ Departments of Philosophy, the CUNY Graduate Center, \\ 365 5th Ave, New York, NY 10016, USA. \\ and the University of Melbourne, \\ Grattan Str., Parkville, Victoria, 3010, Australia. \\ E-mail: priest.graham@gmail.com
}

\begin{abstract}
Given a formal language, a metalanguage is a language which can express amongst other things - statements about it and its properties. And a metatheory is a theory couched in that language concerning how some of those notions behave. Two such notions that have been of particular interest to modern logicians - for obvious reasons are truth and validity. These notions are, however, notoriously deeply entangled in paradox. A standard move is to take the metalanguage to be distinct from the language in question, and so avoid the paradoxes. One of the attractions of a dialetheic approach to the paradoxes of self-reference is that this move may be avoided. One may have a language with the expressive power to talk about - among other things - itself, and a theory in that language about how notions such as truth and validity for that language behave. The contradictions delivered by these notions are forthcoming, but they are quarantined by the use of a paraconsistent logic. The point of this paper is to discuss this project, the extent to which it has been successful, and the places where issues still remain.
\end{abstract}

Keywords: truth, validity, dialetheism, paraconsistency, paradox, self-reference, model theory, material detachment, set theory

For citation: Priest G. "Metatheory and dialetheism", Logicheskie Issledovaniya / Logical Investigations, 2020, Vol. 26, No. 1, pp. 48-59. DOI: 10.21146/2074-1472-2020-26-1-48-59

Dedication: Sasha Karpenko was an outstanding logician, and a key player in logic in Russia. I met him on a couple of trips to Moscow. He was an engaging philosophical interlocutor, and a kind and warm host. I dedicate this paper fondly to his memory.

\section{Introduction}

Logic is about arguments. Arguments are expressed in languages; and for modern logicians, these are formal languages. For such a language, a metalanguage is a language which can express - amongst other things - statements about that language and its properties. And a metatheory is a theory couched 
in that language concerning how some of those notions behave. Two such notions that have been of particular interest to modern logicians - for obvious reasons - are truth and validity. These notions are notoriously, however, deeply entangled in paradox. A standard move, since Tarski's Warheitsbegri Tarski, 1935 is to take the metalanguage to be distinct from language in question, and so avoid the paradoxes.

I think that most logicians would now agree with Tarski that this move is just an artifice - one with little justification other than to avoid contradiction. Natural languages, in which, of course, such paradoxes find their home, most certainly do not seem to be structured in this hierarchical fashion.

One of the attractions of a dialetheic approach to the paradoxes of selfreference is that this move may be avoided. One may have a language with the expressive power to talk about - among other things - itself, and a theory in that language about how notions such as truth and validity for that language behave. The contradictions delivered by these notions are forthcoming, but they are quarantined by the use of a paraconsistent logic.

The point of this paper is to discuss this project, the extent to which it has been successful, and the places where issues still remain. In the first part of the paper I will discuss truth; in the second and much longer part, I will discuss validity.

\section{Truth}

As far as truth is concerned, what we need is a language which contains a way of referring to its own sentences, and a truth predicate that applies to these. As is now standard, a simple way of talking about sentences is to suppose that our theory contains arithmetic, and use a gödel coding. I will assume this in what follows. In particular, given any sentence, $A$, of the language, I will write $\langle A\rangle$ for the numeral of the gödel code of $A$. This is its name.

The theory must also tell us how truth behaves. Given that avoiding paradox is no longer necessary, the natural and obvious thought is that it should deliver all instances of the $T$-schema:

- $T\langle A\rangle$ iff $A$

There is a question about how to understand the 'iff' here, and there are various possibilities. One is as the biconditional of some relevant logic, $\leftrightarrow$; another is as bi-deducibility, $\dashv$; another is as a material biconditional, $\equiv$ (where $A \equiv B$ is $(\neg A \vee B) \wedge(\neg B \vee A))$. Given some simple assumptions, the first of these options is the strongest. In particular, any results about what cannot be proved using this notion of conditionality carry over to the weaker notions. So let me discuss this option. 
Take the logic of our theory to be an appropriate relevant logic; ${ }^{1}$ and take a theory in this logic which contains enough arithmetic, plus the axiom schema:

- $T\langle A\rangle \leftrightarrow A$

for any closed sentence, $A$. It is now well known that such a theory is inconsistent but non-trivial. Thus, one can show that some sentences are both true and not true - for example, the sentence $L$ of the form $\neg T\langle L\rangle$. However, one cannot prove everything. In particular, any sentence in the $\rightarrow$-free fragment in the language which is grounded in Kripke's sense Kripke, 1975 behaves consistently. The proof of this and references may be found in Priest, 2008, $\S 8]$.

Of course, one may wish for more from a theory of truth than this. In particular, Tarski showed how to give a theory of truth in which truth conditions are given recursively, and the T-Scheme is then proved. One can do this too. What is essentially the Tarski construction can also be carried out in a paraconsistent logic. The details of the construction can be found in Priest, 1987, ch. 9].

Before we move on to validity, let me make a couple of comments on two notions cognate with truth: satisfaction and denotation. The construction which shows the non-triviality of the $T$-Schema may be used to establish the non-triviality of the (one-place) Satisfaction-Schema: ${ }^{2}$

- $y S\langle A\rangle \leftrightarrow A_{x}(y)$

where $S$ is the satisfaction predicate, $A$ is any formula of one free variable, $x$, and $A_{x}(y)$ is the result of substituting $y$ for $x$ in $A$ (relabelling bound variables if necessary to avoid a clash).

We may define the denotation predicate, $D$, in the obvious way: $\langle t\rangle D y:=$ $y S\langle x=t\rangle$, where $t$ is any (closed) term, to deliver the Denotation Schema:

- $\langle t\rangle D y \leftrightarrow y=t$

The same results then follow for denotation.

There is an extra complication in this case, however. I have tacitly assumed that the language we are dealing with till now does not contain descriptive terms. If we add such terms to the language, complications arise in the case of denotation. Non-triviality results are obtainable, at least when the

\footnotetext{
${ }^{1}$ Exactly what this is, we do not need to go into here. But I assume that the logic contains the Principle of Excluded Middle, $\models A \vee \neg A$, but not Absorption, $A \rightarrow(A \rightarrow B) \models A \rightarrow B$. A suitable such logic is $B X$. See Priest, 2008, 10.4a.12].

${ }^{2}$ And its generalisation to an arbitrary number of free variables.
} 
biconditional of the Scheme is bi-deducibility. ${ }^{3}$ However, matters are less than straightforward, since they are entangled with assumptions one makes about denotation.

\section{Validity: Preliminary Considerations}

Let us now move from truth to the somewhat move vexed notion of validity; and let us start by getting some relatively straightforward matters out of the way.

In modern logic, validity can be defined syntactically, in terms of some proof system, or semantically, in terms of interpretations of the language. Any axiomatic theory that contains arithmetic can define its own syntactic validity relation, at least for finite premise-sets. Thus, given an axiom system for the theory, whose axioms are the members of some decidable set $X$, then if $Y$ is finite set of sentences, $Y \vdash A$ iff there is a finite sequence of formulas, $A_{1}, \ldots, A_{n}$ such that $A$ is $A_{n}$, and for any $i<n, A_{i}$ is either in $X$, or in $Y$, or follows from some sentences earlier in the sequence by some rule of inference. All this can be expressed in arithmetic in a familiar fashion. Nothing about paraconsistency changes this matter.

Of course, if the arithmetic theory is axiomatic and consistent, it cannot prove its own consistency. On the other hand, there are complete and inconsistent arithmetics based on a paraconsistent logic which can prove their own non-triviality. ${ }^{4}$

So let us switch our attention to a semantic definition of validity. According to such a definition, $X \models A$ iff every interpretation (appropriate for the logic in question) which makes all the members of $X$ true makes $A$ true. First, note that the notion of truth here is truth-in-an-interpretation, not truth simpliciter. Of course, one might hope that there is some interpretation such that truth in that interpretation is extensionally equivalent to truth simpliciter (a standard interpretation); but such is not required for a definition of validity. Next, note the the notion of an interpretation, as it is standardly understood, is a set-theoretic one. (An interpretation comprises a domain of quantification, a denotation function, etc.) Hence, to give such a definition requires one to deploy the language of set theory.

How to do so is familiar to anyone with a knowledge of the elements of model-theory. Thus, suppose our language is that of first-order set-theory, and our theory is $Z F C$. Then, given any language, $L$, and a notion of interpretation for that language, we can define the relation $X \models A$ in a straightforward fashion. In particular, $L$ can be the language of first-order set theory, and the

\footnotetext{
${ }^{3}$ See Priest, 1998, Priest, 1999 and Priest, 2005, ch. 8].

${ }^{4}$ See Priest, 1987, 17.4].
} 
notion of interpretation can be that of classical logic. The notion of validity defined can then be the one deployed in $Z F C$. In that sense, $Z F C$ can define its own validity relation.

What it cannot do, at least if $Z F C$ is consistent, is to prove that there is a standard model. That is, it cannot establish the existence of an interpretation, $I$, such for any sentence, $A$, in the language of set theory, $\langle A\rangle$ is true in $I$ iff $A$. This fact deprives us of a rationale as to why one may legitimately deploy this notion of validity when reasoning in $Z F C$ itself - where we are, presumably, interested in deploying a notion of validity that preserves truth simpliciter. It is not at all obvious how to address this issue. Probably the best known approach is to apply the notion of informal rigour, as suggested by [Kreisel, 1967].

\section{Paraconsisent Validity}

Having got these matters straight, let us now turn to the issue of a modeltheoretic definition of validity appropriate for a dialetheic solution to the paradoxes of self-reference using a paraconsistent logic.

Of course, if one holds that $Z F C$ is the correct set theory, matters are exactly the same as in the case of classical logic. One simply replaces the notion of a classical interpretation with that of the notion of interpretation appropriate for the paraconsistent logic at hand. This approach is hardly available to someone who endorses a dialetheic solution to the paradoxes of set-theory, however. For in such an approach one endorses the naive comprehension schema:

- $\exists x \forall y(y \in x$ iff $A)$

where $A$ is arbitrary. ${ }^{5}$ The set-theoretic paradoxes are then forthcoming, but they are quarantined by the use of the paraconsistent logic. Naturally, this commits one to a set-theory quite different from $Z F C$.

And here we meet our first real problem. What is that set theory? The matter turns again on how one is to understand the conditional 'iff' in the schema. There are presently two approaches to the problem. The first is to take the underlying logic of the theory to be an appropriate relevant logic, and take the biconditional to be that of this logic. This approach has been developed at greatest length by Weber Weber, 2010; Weber, 2012, who has shown how to prove most of the standard results concerning ordinal and cardinal arithmetic (and many other interesting things) in this theory. The theory is also known to be non-trivial, due to a proof of Brady. ${ }^{6}$ It is also clear that the

\footnotetext{
${ }^{5}$ Even if one insists that $x$ does not occur free in $A$, the more general case follows. A proof of this for set theory based on a substructural logic can be found in Cantini, 2003. Theorem 3.20]. The same proof works in relevant logic.

${ }^{6}$ For discussion and references, see, again, Priest, 2002, § 8].
} 
standard model-theoretic definition of validity can be given for the logic used. One simply defines it and the notions it requires in the obvious way. One has at one's disposal, after all, the naive comprehension schema. Unfortunately, it is not known how much of standard model theory can be established in this way, since the usual proofs deploy inferences not available in the theory. Naturally, the definitions themselves are not much good unless we can show that the notions defined have at least some minimal properties, such as that the proof system of the logic is sound with respect to the notion of validity. Until more is known about these matters, it is impossible to say anything much for the issue at hand. ${ }^{7}$

A quite different approach to paraconsistent set theory is to take its underlying logic to be that of the paraconsistent logic $L P$, and to take the biconditional in the comprehension scheme to be the material biconditional of that logic. ${ }^{8}$ If one takes this approach then, since the conditional of this logic does not detach, one cannot prove anything much from the set theoretic axioms. A different approach is required. This is itself model-theoretic. It can be shown that there are interpretations of the language of set-theory which are models of both the naive comprehension schema and all the theorems of $Z F C$. The set of sentences true in such models is inconsistent, since one can prove paradoxes such as Russell's, but non-trivial. And now, if one may assume that the universe of sets - or universes of sets if there is more than one - is/are given by such (an) interpretation(s), then one may simply help oneself to anything that can be engineered in $Z F C$, including the definition of validity for the logic $L P$, and all those results about it that may be proved. ${ }^{9}$

One thing that this approach can do, which can not be done classically, to deliver us a standard model. For it can be shown that there are interpretations of the kind just indicated in which all instances of the following are true:

- $\exists x\left(x\right.$ is an $L P$ interpretation $\left.\wedge\left(A \equiv\left(x \Vdash^{+}\langle A\rangle\right)\right)\right)$

\footnotetext{
${ }^{7}$ In Weber, 2016], Weber shows one way in which semantic validity may be defined for propositional logic, and proves soundness and completeness. His approach comes with a steep downside, however: every inference is invalid (though some are valid too). Non-triviality proofs also become trivially easy, and so somewhat vacuous. It seems to me that many of the problems arise because Weber endorses the exclusivity of truth and falsity in an interpretation (p. 539). This assumption, it seems to me, could be jettisoned. However, this is not the place to go into that matter.

${ }^{8}$ This account of set-theory is proposed in the second edition of [Priest, 1987, ch. 18], and explored at greater length in Priest, 2017, §§ 10-12].

${ }^{9}$ Naturally, one may ask why such an assumption is justified. Perhaps there is no better answer than that it seems to validate the things we take to be true of sets - though of course it does not justify these beliefs.
} 
(Here, $\Vdash^{+}$denotes truth in a $L P$ interpretation.) Assuming, again, that the universe(s) of sets is (are) like this then justifies the application of this notion of validity in reasoning about sets. Of course, one might reject the claim that the "intended" models of set theory do contain a standard model. In that case, we would be no worse off than in the classical case, and we would have to deploy some strategy such as Kreisel's in an attempt to justify using $L P$ to reason about sets.

A second positive fact about this notion of validity, is that it solves a version of the validity-Curry paradox, first proposed by [Beall, Murzi, 2013]. Let us write $V(\langle A\rangle,\langle B\rangle)$ to express the fact that the inference from $A$ to $B$ is valid. By standard techniques of self-reference, we can construct a sentence, $D$, of the form $V(\langle D\rangle,\langle\perp\rangle)$, where $\perp$ is a logical constant such that, in $L P, \perp \models A$, for any $A$. Then there is a natural argument for $\perp$. This argument fails in the models we are dealing with, since they are closed under $L P$ consequence, and they are not trivial. Where the argument breaks down may depend on exactly how it is formulated; but essentially, it fails due to the invalidity of material detachment. ${ }^{10}$

\section{Validity and Detachment}

In the last couple of sections, I will take up two issues arising from this account of validity. In what follows, I will stick to the one-premise case of validity for simplicity. The considerations clearly carry over the general case.

The first issue concerns the fact that if we define validity in the way described, the connection between the premises of a valid inference and its conclusion is only a material one. ${ }^{11}$ The definition of validity has the following form: ${ }^{12}$

$$
\text { - } \forall I\left(I \Vdash^{+} A \supset I \Vdash^{+} B\right)
$$

Even though an inference is valid, then, the move from $I \Vdash^{+} A$ to $I \Vdash^{+} B$ is not valid in $L P$. And if $I$ is the standard model, the same goes for truth simpliciter. This does not mean that no inference from the first to the second is possible. Failure of detachment occurs in $L P$ only when the antecedent of the conditional is both true and false. Hence the move is legitimate provided that this is not the case. This observation can be built into a formal non-monotonic logic, $L P m$, in which the inference from $C$ and $C \supset D$ to $D$ is a valid default

\footnotetext{
${ }^{10}$ For further discussion, see [Priest, 2017, § 4.2].

${ }^{11}$ The following comes from Priest, 2017, § 14].

${ }^{12} \vdash^{+}$is a relationship between an interpretation and a sentence, so it would be more correct to write the relation as $I \Vdash^{+}\langle A\rangle$. However, here and in what follows I omit the quotation marks, as logicians usually do.
} 
inference. ${ }^{13}$ Using $L P m$, we can then move from $I \Vdash^{+} A$ to $I \Vdash^{+} B$ provided that we do not have $I \|^{+} A$ as well (which is quite different from $I \Vdash^{+} \neg A$ ).

However, it remains the case that this is a default inference. What is the significance of this? This depends on how one understands the model-theory. A straightforward way to understand the model-theoretic definition of validity is as specifying the meaning of 'valid'. In this case, even valid deductive inferences are, in the last instance, default inferences.

It might be thought odd to have the validity of a deductive inference grounded in a defeasible inference. But a little thought may assuage this worry. The difference between a material $I \Vdash^{+} A \supset I \Vdash^{+} B$ and a detachable $I \Vdash^{+} A \rightarrow I \Vdash^{+} B$ is not as great as might be thought. Both are simply true (or false) statements. Inference, by contrast, is an action. Given the premises of an argument, an inference is a jump to a new state. No number of truths is the same thing as a jump. (This is the moral of Lewis Carroll's celebrated dialogue between Achilles and the Tortoise [Carroll, 1895].) None the less, truths of a certain kind may ground the jump, in the sense of making it a reasonable action. There is no reason why a sentence of the form $C \supset D$ may not do this, just as much as one of the form $C \rightarrow D$. It is just that one of the latter kind always does so, while one of the former kind does so only sometimes (normally).

If it is not clear how a defeasible warrant for an action can work, merely consider sentences of the form:

(*) You promised to do $x$.

The truth of $\left(^{*}\right)$ is normally a ground for doing $x$, in the sense of making it reasonable to do so. But, to use a celebrated example, suppose that $\left(^{*}\right)$ is true, where the $x$ in question is the returning of a weapon to a certain person. And suppose that the person comes requesting the weapon, but you know that they intend to use it to commit murder. Then the truth of $(*)$ does not, in this context, ground the action. So with validity and the material conditional: the truth of a sentence of a certain kind may ground an appropriate action in normal circumstances, but fail to do so in unusual circumstances.

Another way to take the model-theoretic account of validity is as providing, not the meaning of 'valid', but merely an extensional characterisation of what is valid. The meaning of 'valid' itself can be characterised in a different way, say proof-theoretically (or simply taken as an indefinable primitive). The modeltheoretic account merely gives us a characterisation of what inferences are or are not deductively valid - nothing more. Valid inferences can then simply license detachment of their conclusions, though this aspect of things may not be captured by the characterisation. In a similar way, an inferentialist who

\footnotetext{
${ }^{13}$ See the second edition of $[$ Priest, 1987, ch. 16].
} 
takes validity to be defined in terms of the meanings of the logical constants, spelled out in terms of introduction and elimination rules, may yet hold that a model-theoretic definition of validity delivers an extensionally equivalent characterisation (if sound and complete), though this may miss aspects of validity itself.

\section{Dialetheic Validity}

The second issue I will take up concerns the extent to which validity is itself a dialetheic notion. Let us suppose that we are working within one of the models of the kind we saw to exist in $\S 4$; and let us suppose that the model does verify the existence of a standard model.

The following argument is due, in effect, to Young Young, 2005. Let $M$ be the standard model. Then given the resources of self-reference, we can find a sentence, $D$, of the form $M \|^{+} D$. The facts about the standard model then deliver:

- $M \Vdash^{+} D \equiv M \nvdash^{+} D$

It follows in $L P$ that $M \Vdash^{+} D \wedge M \|^{+} D$. Since $A, \neg B \models \neg(A \supset B)$, it also follows that

$$
\text { - } \neg\left(M \Vdash^{+} D \supset M \Vdash^{+} D\right)
$$

So $\exists x \neg\left(x \Vdash^{+} D \quad \supset \quad x \Vdash^{+} D\right)$, i.e., $\neg \forall x\left(x \Vdash^{+} D \quad \supset \quad x \Vdash^{+} D\right)$. That is, the inference from $D$ to $D$ is invalid. It follows that $p \not \models p$, since $p$ has an invalid substitution instance - even though $p=p$ as well. Perhaps this is not surprising. Truth is intimately connected with validity - at least when we have a standard model around. So one might expect self-referential constructions to deliver inconsistencies concerning validity; and the inference from $p$ to $p$ is not a terribly useful one!

It might be thought that Young's argument can be extended to establish that other valid inferences are also invalid. Thus, consider, for example, the inference from $p \wedge q$ to $p$. If we could show that:

- [1] $\neg\left(M \Vdash^{+} D \wedge D \supset M \Vdash^{+} D\right)$

we would have a similar counter-example. Now, the truth conditions for conjunction give us:

- $I \Vdash^{+} D \wedge D \equiv I \Vdash^{+} D \wedge I \Vdash^{+} D$

or equivalently:

- [2] $I \Vdash^{+} D \wedge D \equiv I \Vdash^{+} D$ 
In $L P$ material equivalents are inter-substitutable, in the sense that $A \equiv B=$ $C(A) \equiv C(B) .{ }^{14}$ So from [2], when $I$ is $M$, we have:

$$
\text { - } \neg\left(M \Vdash^{+} D \supset M \Vdash^{+} D\right) \equiv \neg\left(M \Vdash^{+} D \wedge D \supset M \Vdash^{+} D\right)
$$

But we cannot infer [1] because of the failure of detachment for the material biconditional. The inference is not even a valid default inference, since we know that the left hand side is contradictory. The same sort of problem is going to beset similar extensions of Young's argument to other inferences.

At present, it is not known how inconsistent validity is on the approach under consideration. The natural generalisation of Young's argument does not go through, but that does not mean that there are no others; and at present, there are no arguments which establish that the domain of inconsistency concerning validity is bounded, of the kind that show this for truth. This is, hence, an area where more work is required.

But let us suppose a worst case scenario: every inference is invalid. How damaging a conclusion would this be? Less than one might have thought. (Certainly, much less than a conclusion to the effect that every sentence is not true.) One should remember that every inference is an instance of some formally invalid inference (e.g., $p \vdash q$ ). An inference is acceptable if it is a substitution instance of some formally valid inference. Thus, it is perfectly acceptable to use an inference that is formally valid - it's a substitution instance of itself even if it is invalid too! $!^{15}$

\footnotetext{
${ }^{14}$ See Priest, 2017, 2.3].

${ }^{15}$ I note that there is a way to avoid conclusions to the effect that some valid inferences are invalid, whether they are arguments of Weber's kind or of Young's kind. The definition of semantic validity given in Priest, 1987, 5.2], is slightly different from the one considered above. It uses a truth predicate, and amounts to this:$$
\text { - } A \models B \text { is } \forall I T\left\langle I \Vdash^{+} A \Rightarrow I \Vdash^{+} B\right\rangle
$$

(where $\Rightarrow$ is either $\rightarrow$ or $\supset$, depending on how one thinks of the underlying set theory). Of course, given the $T$-Schema, this makes no difference to what is valid. However, it may well make a difference to what is invalid. One can establish that $\neg\left(M \Vdash^{+} D \Rightarrow M \Vdash^{+} D\right)$, and so $T\left\langle\neg\left(M \Vdash^{+} D \Rightarrow M \Vdash^{+} D\right)\right\rangle$; but one cannot establish that $\neg T\left\langle M \Vdash^{+} D \Rightarrow M \Vdash^{+} D\right\rangle$ unless negation commutes with truth; that is, unless the $T$-schema contraposes. There are reasons to suppose that it does not. See Priest, 1987, 4.6]. A reader of the 1987 text would probably not even have noticed the use of truth in the definition there, or might have supposed its use to be merely stylistic. It would not be unreasonable to do so. Indeed, I myself have ignored this subtlety ever since. However, the use of the truth predicate was not an innocent one: I phrased the definition like this precisely because I thought that arguments of the kind in question might be possible.
} 


\section{Conclusion}

We have now looked at many of the most important aspects of a dialetheic account of metatheory. While it can hardly be claimed that all of these are resolved, the project seems in a more than satisfactory state.

Acknowledgements. Many thanks go to Zach Weber for comments on an earlier draft of this paper. A version was read at the UNILOG conference, Vichy, June 2018.

\section{References}

Beall, Murzi, 2013 - Beall, J., Murzi, J. "Two Flavors of Curry's Paradox", The Journal of Philosophy, 2013, Vol. 110, pp. 143-165.

Cantini, 2003 - Cantini, A. "The Undecidability of Grišin's Set Theory", Studia Logica, 2003, Vol. 74, pp. 345-368.

Carroll, 1895 - Carroll, L. "What the Tortoise Said to Achilles", Mind, 1895, Vol. 4, pp. 278-280.

Kreisel, 1967 - Kreisel, G. "Informal Rigour and Completeness Proofs", in: Problems in the Philosophy of Mathematics, ed. by I. Lakatos, Amsterdam: North Holland, 1967, pp. 138-157.

Kripke, 1975 - Kripke, S. "Outline of a Theory of Truth", Journal of Philosophy, 1975, Vol. 72, pp. 690-716.

Priest, 1987 - Priest, G. In Contradiction, Dordrecht: Martinus Nijhoff; 2nd ed., Oxford: Oxford University Press, 2006.

Priest, 1997 - Priest, G. "On a Paradox of Hilbert and Bernays", Journal of Philosophical Logic, 1997, Vol. 26, pp. 45-56.

Priest, 1998 - Priest, G. "The Trivial Object, and the Non-Triviality of a Semantically Closed Theory with Descriptions", Journal of Applied and Non-Classical Logics, 1998, Vol. 8, pp. 171-183.

Priest, 1999 - Priest, G. "Semantic Closure, Descriptions, and Non-Triviality", Journal of Philosophical Logic, 1999, Vol. 28, pp. 549-558.

Priest, 2002 - Priest, G. "Paraconsistent Logic", in: Handbook of Philosophical Logic, Vol. 6, D. Gabbay, F. Guenthner (eds.), Dordrecht: Kluwer Academic Publishers, 2002, pp. 287-393.

Priest, 2005 - Priest, G. Towards Non-Being, Oxford: Oxford University Press; 2nd ed., 2016.

Priest, 2008 - Priest, G. Introduction to Non-Classical Logic: From If to Is, Cambridge: Cambridge University Press, 2008.

Priest, 2017 - Priest, G. "What If: the Exploration of an Idea", Australasian Journal of Logic, 2017, Vol 14:1, article 1, https://ojs.victoria.ac.nz/ajl/article/ view/4028/3574

Tarski, 1935 - Tarski, A. "Der Wahrheitsbegriff in den Formalisierten Sprachen", Studia Philosophica, 1935, Bd. 1, S. 261-405. Translated as "The Concept of 
Truth in Formalized Languages", in: Logic, Semantics, Metamathematics, ed. by J. Corcoran, 2nd ed., Indianapolis: Hackett, 1983, pp. 152-278.

Weber, 2010 - Weber, Z. "Transfinite Numbers in Paraconsistent Set Theory", Review of Symbolic Logic, 2010, Vol. 3, pp. 71-92.

Weber, 2012 - Weber, Z. "Transfinite Cardinals in Paraconsistent Set Theory", Review of Symbolic Logic, 2012, Vol. 5, pp. 269-293.

Weber, 2016 - Weber, Z. "What is an Inconsistent Truth Table?", Australasian Journal of Philosophy, 2016, Vol. 94, pp. 533-548.

Young, 2005 - Young, G. Revenge: Dialetheism and its Expressive Limitations, $\mathrm{PhD}$ Thesis, University of Glasgow, 2005. 